\title{
Soft sensor modelling by time difference, recursive partial least squares and adaptive model updating
}

DOI:

10.1088/1361-6501/aa57e2

\section{Document Version}

Accepted author manuscript

Link to publication record in Manchester Research Explorer

\section{Citation for published version (APA):}

Fu, Y., Yang, W., Xu, O., Zhou, L., \& Wang, J. (2017). Soft sensor modelling by time difference, recursive partial least squares and adaptive model updating. Measurement Science and Technology, 28(4), [045101]. https://doi.org/10.1088/1361-6501/aa57e2

\section{Published in:}

Measurement Science and Technology

\section{Citing this paper}

Please note that where the full-text provided on Manchester Research Explorer is the Author Accepted Manuscript or Proof version this may differ from the final Published version. If citing, it is advised that you check and use the publisher's definitive version.

\section{General rights}

Copyright and moral rights for the publications made accessible in the Research Explorer are retained by the authors and/or other copyright owners and it is a condition of accessing publications that users recognise and abide by the legal requirements associated with these rights.

\section{Takedown policy}

If you believe that this document breaches copyright please refer to the University of Manchester's Takedown Procedures [http://man.ac.uk/04Y6Bo] or contact uml.scholarlycommunications@manchester.ac.uk providing relevant details, so we can investigate your claim.

\section{OPEN ACCESS}




\title{
Soft sensor modelling by time difference, recursive partial least squares and adaptive model updating
}

\author{
Y Fu ${ }^{1,2}$, W Yang ${ }^{2}, \mathrm{O} \mathrm{Xu}{ }^{1}, \mathrm{~L} \mathrm{Zhou}^{3}, \mathrm{~J} \mathrm{Wang}^{4}$ \\ ${ }^{1}$ Zhijiang College, Zhejiang University of Technology, Hangzhou 310024, China \\ ${ }^{2}$ School of Electrical and Electronic Engineering, University of Manchester, Manchester M13 9PL, UK \\ ${ }^{3}$ School of Automation, Nanjing University of Science and Technology, Nanjing 210094, China \\ ${ }^{4}$ School of Electronic Information and Electrical Engineering, Shanghai Jiaotong University, Shanghai 200240, China \\ E-mail: fuyongfeng@zjut.edu.cn
}

\begin{abstract}
To investigate time-variant and nonlinear characteristics in industrial processes, a soft sensor modelling method based on time difference moving-window recursive partial least square (PLS) and adaptive model updating is proposed. In this method, time difference values of input and output variables are used as training samples to construct the model, which can reduce the effects of the nonlinear characteristic on modelling accuracy and retain the advantages of recursive PLS algorithm. To solve the high updating frequency of the model, a confidence value is introduced, which can be updated adaptively according to the results of the model performance assessment. Once the confidence value is updated, the model can be updated. The proposed method has been used to predict the 4-CBA (i.e. carboxy-benz-aldehyde) content in the PTA (i.e. purified terephthalic acid) oxidation reaction process. The results show that the proposed soft sensor modelling method can reduce computation effectively, improve prediction accuracy by making use of process information and reflects the process characteristics accurately.
\end{abstract}

Key words: Soft sensor, Time difference, Recursive PLS, Model updating

\section{List of symbols}

\section{$\boldsymbol{B}$}

$c_{h} \quad$ Standardised feature vector

$\boldsymbol{e} \quad$ Noise vector

$\boldsymbol{E} \quad$ Residual matrix

$\boldsymbol{F} \quad$ Residual matrix

$l \quad$ Number of output variables

$m \quad$ Number of input variables

$n \quad$ Number of available samples

$N \quad$ Window length

$\boldsymbol{p}_{h} \quad$ Loading vector of the $h$ th latent variable

$\boldsymbol{P} \quad$ Loading matrix

$\boldsymbol{q}_{h} \quad$ Loading vector of the $h$ th latent variable

$Q \quad$ Loading matrix

$\boldsymbol{R} \quad$ Error matrix

$\boldsymbol{t}_{h} \quad$ Score vector of $h$ th latent variable

$\begin{array}{ll}- & \text { Mean of the } i \text { th sample in the data window of length } N \\ x_{i, N} & \text { Online updated mean } \\ x_{i, N+1} & \text { Auxiliary variable values of new data } \\ \boldsymbol{x}\left(t^{\prime}\right) & \\ \Delta \boldsymbol{x}\left(t^{\prime}\right) & \text { Time difference values of } \boldsymbol{x}\left(t^{\prime}\right) \\ \boldsymbol{X} & \text { Input data matrix } \\ \boldsymbol{X}(t) & \text { Present values of auxiliary variables } \\ \boldsymbol{X}(t-i) & \text { Values in some time } i \text { before } \boldsymbol{X}(t) \\ \Delta \boldsymbol{X}(t) & \text { Time difference values of } \boldsymbol{X}(t) \\ \boldsymbol{y}(t) & \text { Present values of objective variables } \\ \boldsymbol{y}(t-i) & \text { Values in some time } i \text { before } \boldsymbol{y}(t) \\ \Delta \boldsymbol{y}(t) & \text { Time difference values of } \boldsymbol{y}(t) \\ y\left(t^{\prime}\right) & \text { Predicted objective variable of new data } \\ \Delta y\left(t^{\prime}\right) & \text { Time difference value of } y\left(t^{\prime}\right) \\ \boldsymbol{Y} & \text { Output data matrix } \\ \boldsymbol{\beta} & \text { Regression coefficient matrix } \\ \hat{\boldsymbol{\beta}} & \text { Estimate of } \boldsymbol{\beta}\end{array}$




$\begin{array}{ll}\boldsymbol{T} & \text { Score matrix } \\ \boldsymbol{u}_{h} & \text { Score vector of } h \text { th latent variable } \\ \boldsymbol{U} & \text { Score matrix } \\ \boldsymbol{w}_{h} & \text { Standardised feature vector }\end{array}$

$\begin{array}{ll}\delta & \text { Confidence limit } \\ \delta_{e} & \text { Adaptive confidence limit } \\ \sigma_{i, N}^{2} & \text { Variance of } i \text { th sample in data window of length } N \\ \sigma_{i, N+1}^{2} & \text { Online updated variance }\end{array}$

\section{Introduction}

In industry, some process variables are closely related to the product quality. However, in many cases, it is difficult to measure those variables online for technical or economic reasons. Therefore it is difficult to monitor and control the processes in real time, the product quality cannot be ensured. To address this issue, soft sensors have been developed in recent years and used to estimate the process variables. With a soft sensor, an inferential model can be constructed between some variables that can be measured online (also called auxiliary variables) and other variables that cannot be measured online (also called objective variables). Then an objective variable can be estimated using the model. If the objective variables can be estimated accurately by the use of soft sensors, optimal control of those industrial processes can be implemented [1-3].

Among various soft sensor modelling methods, the partial least squares (PLS) method is most popular because it is simple in calculation, not very sensitive to noise and can provide good correlation between the measured variables (i.e. the input variables) and the estimated variables (i.e. the output variables) [4-6]. However, the PLS method can only reflect a linear relationship between the input and output variables. Considering that industrial processes in general have time-varying characteristics, the prediction accuracy of the soft sensors based on PLS would gradually decrease due to various reasons, e.g. the changes in the state of a process, sensor drift, and loss of catalysing performance.

To overcome the problem that the PLS method does not reflect the non-linear relationship between the input and output variables, Rosipal et al. proposed the Kernel PLS method [7-8]. While this method can overcome to some extend the shortcomings of PLS, its non-linear approximation depends largely on the complexity of the model, and its calculation is complicated. To reduce the degradation caused by the change in a process, Helland et al. proposed a recursive PLS algorithm, which can update the parameters of the PLS model online [9]. Qin modified and supplemented the recursive PLS algorithm, and used this method to model and estimate some chemical processes [10]. A recursive algorithm can make use of information from old and new data, and can effectively track the dynamic characteristics of a process. The problem with a recursive algorithm is that a large number of repetitive calculations are needed and with the continuous increase in the number of modelling samples, there is a data saturation problem. In addition, too many old samples hide the information of new samples.

Moving window [10] or forgetting factor $[5,11]$ is an effective way to solve these problems. However, a moving window method treats new data and old data equally in model updating. Therefore, it cannot keep a good track of the dynamic characteristics of an industrial process. As for the forgetting factor, it is difficult to be set or optimised. Mu et al. proposed a moving-window recursive PLS algorithm that combines a moving-window and a recursive algorithm [12]. This algorithm can modify the mean and variance online, which 
enables the method to keep the old sample information and effectively track the dynamic characteristics of a process. However, every time a new sample is collected, the model needs to be updated. The updating rate is therefore high, resulting in low computational efficiency [13].

A data block-based recursive PLS method combines the moving-window and forgetting factor [5]. In this method a model is updated only when new data are accumulated to a fixed length. Although this method can reduce the model updating rate, the data saturation problem is still remaining and it is difficult to set the forgetting factor. In addition, the length of the data blocks can only be set by experience because there is no established rule to follow.

To address the above issues, we propose a soft sensor modelling method based on time difference, moving-window, recursive PLS and adaptive model updating. This method firstly uses the time difference values between the current sample data and the previous sample data to build a moving-window recursive PLS model. This time difference moving-window recursive PLS model not only can fully retain the good characteristics of linear PLS model, and improve the model's approximation accuracy to the nonlinear characteristics of the industrial process. Then, this method can be automatically generated model confidence limit based on the initial characteristics of the process. This confidence limit can update adaptively with the time-varying nature of the process and the results of the model performance assessment. If the model prediction error is greater than the confidence limit, the model will update. Once the model updated, the confidence limit value will update too. The proposed method is applied to build a soft sensor model based on actual industrial process data to predict the 4-CBA content in PTA oxidation process. The results indicate that the method is effective. It can reduce computation and improve the prediction accuracy effectively.

\section{Basic algorithms}

\subsection{PLS algorithm}

Let's consider a pair of input data matrix, $\quad \boldsymbol{X} \in \boldsymbol{R}^{n \times m}$, and an output data matrix $\boldsymbol{Y} \in \boldsymbol{R}^{n \times l}$, which have been centralised and normalised, with $n$ being the number of available samples, $m$ the number of input variables, and $l$ the number of output variables. The relationship between $\boldsymbol{X}$ and $\boldsymbol{Y}$ can be represented by

$$
\boldsymbol{Y}=\boldsymbol{X} \boldsymbol{\beta}+\boldsymbol{e}
$$

where $\boldsymbol{\beta}$ is the coefficient matrix and $\boldsymbol{e}$ is the noise vector.

A PLS algorithm decomposes matrixes $\boldsymbol{X}$ and $\boldsymbol{Y}$ into two external relations, called external model:

$$
\begin{gathered}
\boldsymbol{X}=\boldsymbol{T} \boldsymbol{P}^{T}+\boldsymbol{E}=\sum_{h=1}^{a} \boldsymbol{t}_{h} \boldsymbol{p}_{h}^{T}+\boldsymbol{E} \\
\boldsymbol{Y}=\boldsymbol{U} \boldsymbol{Q}^{T}+\boldsymbol{F}=\sum_{h=1}^{a} \boldsymbol{u}_{h} \boldsymbol{q}_{h}^{T}+\boldsymbol{F}
\end{gathered}
$$

where $\boldsymbol{T}=\left[\boldsymbol{t}_{1}, \boldsymbol{t}_{2}, \cdots, \boldsymbol{t}_{a}\right]$ and $\boldsymbol{U}=\left[\boldsymbol{u}_{1}, \boldsymbol{u}_{2}, \cdots, \boldsymbol{u}_{a}\right]$ are called score matrix, $\boldsymbol{P}=\left[\boldsymbol{p}_{1}, \boldsymbol{p}_{2}, \cdots, \boldsymbol{p}_{a}\right]$ and $\boldsymbol{Q}=\left[\boldsymbol{q}_{1}, \boldsymbol{q}_{2}, \cdots, \boldsymbol{q}_{a}\right]$ are called loading matrix, $\boldsymbol{E}$ and $\boldsymbol{F}$ are corresponding residual matrix, $a$ is the number 
of latent variables, $\boldsymbol{t}_{h}$ and $\boldsymbol{u}_{h}$ are the score vectors of $h$ th latent variable, and $\boldsymbol{p}_{h}$ and $\boldsymbol{q}_{h}$ are the corresponding loading vectors.

Score vectors $\boldsymbol{t}_{h}$ and $\boldsymbol{u}_{h}$ can be solved by

$$
\boldsymbol{t}_{h}=\boldsymbol{E}_{h-1} \boldsymbol{w}_{h}, \quad \boldsymbol{u}_{h}=\boldsymbol{F}_{h-1} \boldsymbol{c}_{h}
$$

where $\boldsymbol{E}_{h}=\boldsymbol{E}_{h-1}-\boldsymbol{t}_{h} \boldsymbol{p}_{h}^{T}(h=1,2, \cdots, a), \quad \boldsymbol{E}_{0}=\boldsymbol{X} ; \boldsymbol{F}_{h}=\boldsymbol{F}_{h-1}-\boldsymbol{u}_{h} \boldsymbol{q}_{h}^{T}(h=1,2, \cdots, a), \quad \boldsymbol{F}_{0}=\boldsymbol{Y} ; \quad \boldsymbol{w}_{h}$ and $\boldsymbol{c}_{h}$ are the standardised feature vectors corresponding to the main characteristic values of $\boldsymbol{E}_{h-1}^{T} \boldsymbol{F}_{h-1} \boldsymbol{F}_{h-1}^{T} \boldsymbol{E}_{h-1}$ and $\boldsymbol{F}_{h-1}^{T} \boldsymbol{E}_{h-1} \boldsymbol{E}_{h-1}^{T} \boldsymbol{F}_{h-1}$ respectively.

The relationship between $\boldsymbol{U}$ and $\boldsymbol{T}$ is called the internal model, which can be expressed as

$$
\boldsymbol{U}=\boldsymbol{T} \boldsymbol{B}+\boldsymbol{R}=\sum_{h=1}^{a} \boldsymbol{b}_{h} \boldsymbol{t}_{h}+\boldsymbol{R}
$$

where $\boldsymbol{B}=\operatorname{diag}\left[b_{1}, b_{2}, \cdots, b_{a}\right]$ is the regression coefficient matrix, which is determined by the minimised error matrix $\boldsymbol{R}$.

In a PLS algorithm, the estimate $\hat{\boldsymbol{\beta}}$ of the regression coefficient matrix $\boldsymbol{\beta}$ is

$$
\hat{\boldsymbol{\beta}}=\overline{\boldsymbol{W}} \boldsymbol{B} \boldsymbol{Q}^{T}
$$

where $\overline{\boldsymbol{W}}=\left[\overline{\boldsymbol{w}_{1}}, \overline{\boldsymbol{w}_{2}}, \cdots, \overline{\boldsymbol{w}_{a}}\right], \overline{\boldsymbol{w}_{1}}=\boldsymbol{w}_{1}, \overline{\boldsymbol{w}_{k}}=\prod_{h=1}^{k-1}\left(\boldsymbol{I}_{h}-\overline{\boldsymbol{w}_{h}} \boldsymbol{p}_{h}^{T}\right), \quad k=2, \cdots, a$.

The detailed description of the PLS algorithm can be found in references [9-10, 14].

\subsection{Time difference algorithm}

In a traditional soft sensor modelling method, when the auxiliary variables set $\boldsymbol{X}(t)$ and the objective variables set $\boldsymbol{y}(t)$ are given, the relationship between $\boldsymbol{X}(t)$ and $\boldsymbol{y}(t)$ can be found using a regression method.

In a time difference modelling method $[15,16]$, the time difference values $\Delta \boldsymbol{X}(t)$ and $\Delta y(t)$ of $\boldsymbol{X}(t)$ and $\boldsymbol{y}(t)$ are firstly calculated by

$$
\begin{gathered}
\Delta \boldsymbol{X}(t)=\boldsymbol{X}(t)-\boldsymbol{X}(t-i) \\
\Delta \boldsymbol{y}(t)=\boldsymbol{y}(t)-\boldsymbol{y}(t-i)
\end{gathered}
$$

where $\boldsymbol{X}(t)$ and $\boldsymbol{y}(t)$ are present values of auxiliary variables and objective variable respectively, and $\boldsymbol{X}(t-i)$ and $\boldsymbol{y}(t-i)$ are values in some time $i$ before the present time.

The relationship between $\Delta \boldsymbol{X}(t)$ and $\Delta \boldsymbol{y}(t)$ can be modelled by regression methods. In the prediction process, when new data $\boldsymbol{x}\left(t^{\prime}\right)$ are collected, the time difference value $\Delta \boldsymbol{x}\left(t^{\prime}\right)$ of the new data is firstly calculated by 


$$
\Delta x\left(t^{\prime}\right)=x\left(t^{\prime}\right)-x\left(t^{\prime}-i\right)
$$

Using the time difference $\Delta x\left(t^{\prime}\right)$, the constructed model can predict the time difference value of $y\left(t^{\prime}\right)$, $\Delta y\left(t^{\prime}\right)$. Then $y\left(t^{\prime}\right)$ can be calculated by

$$
y\left(t^{\prime}\right)=\Delta y\left(t^{\prime}\right)+y\left(t^{\prime}-i\right)
$$

because $y\left(t^{\prime}-i\right)$ is given previously.

\subsection{Time difference PLS algorithm}

The PLS algorithm has many advantages. It is simple and has clear physical meaning. It can effectively overcome the collinearity problems and can make the model contain the minimum number of independent variables. A problem with the PLS algorithm is that it is essentially a linear regression method, but industrial processes are often non-linear, thus the prediction accuracy of the model built directly by a PLS algorithm is not high. In this paper, the above-mentioned time difference algorithm is used to build a soft sensor model, i.e. instead of directly using the auxiliary variables set $\boldsymbol{X}(t)$ and the objective variables set $\boldsymbol{y}(t)$, their time difference values $\Delta \boldsymbol{X}(t)$ and $\Delta \boldsymbol{y}(t)$ are used to build a soft sensor model. The principle is as follows.

Suppose a nonlinear process model as follows.

$$
y=f(\boldsymbol{x})
$$

At any point $\boldsymbol{x}_{0}=\left[x_{10}, x_{20}, \cdots, x_{m 0}\right]$, using Taylor's expansion and omitting the higher-order terms, the following equation can be obtained,

$$
f(\boldsymbol{x})=f\left(x_{0}\right)+\left.\left[\frac{\partial f}{\partial x_{1}}\left(x_{1}-x_{10}\right)+\cdots+\frac{\partial f}{\partial x_{m}}\left(x_{m}-x_{m 0}\right)\right]\right|_{x=x_{0}}
$$

and

$$
\Delta y=f(\boldsymbol{x})-f\left(x_{0}\right)=\left.\left[\frac{\partial f}{\partial x_{1}} \Delta x_{1}+\frac{\partial f}{\partial x_{2}} \Delta x_{2}+\cdots+\frac{\partial f}{\partial x_{m}} \Delta x_{m}\right]\right|_{x=x_{0}}
$$

According to the above derivation, the relationship between $\Delta y$ and $\Delta x$ is linear. Thus the PLS model built by the time difference values $\Delta y$ and $\Delta x$ has good nonlinear regression performance, and can improve the prediction accuracy [17]. Furthermore, because the time-difference PLS algorithm uses time difference values of the input and output data to build a soft sensor model, slow changes in an industrial process will not have significant impact on the prediction accuracy.

\section{Recursive PLS algorithm and adaptive online updating}

As described above, the time difference PLS algorithm has good nonlinear regression performance. When the production process has strong time-varying characteristics, or the operating point changes frequently, however, the prediction accuracy will gradually decrease. To reduce the degradation of time difference PLS 
model, it is necessary to update the model online [18].

The moving-window recursive algorithm has been widely used because it can effectively track the dynamic characteristics of a process and solve the "data saturation" problem. However, the updating rate of the ordinary moving-window model is high, leading to a heavy computational load and reduced real-time performance. Considering the above issues, an adaptive updating strategy is used in this paper. First, a confidence limit is set according to the initial characteristics of the process. Then the confidence limit is updated adaptively with the dynamic change in the process and the prediction results. When the prediction error is bigger than the confidence limit, the model parameters will be updated and hence the confidence limit is updated. Otherwise, the model will not be updated.

\subsection{Moving-window recursive PLS algorithm based on variance and mean online updating}

Because the PLS algorithm can be used for modelling high-dimensional data with few samples, the data length generally used for modelling is not large, with the ordinary moving-window recursive method used. When a new sample is collected, the oldest sample will be abandoned, which may lose information. In the moving-window recursive algorithm based on online updating of variance and mean, the variance and mean will be updated online, when a new sample is collected. Thus, part of information of old data can be retained in the model through variance and mean. Then, the oldest sample will be discarded, keeping constant modelling sample length $N$.

Online recursive equations of the mean and the variance are given by

$$
\begin{gathered}
\bar{x}_{i, N+1}=\frac{N}{N+1} \bar{x}_{i, N}+\frac{1}{N+1} x_{i, N+1} \\
\sigma_{i, N+1}^{2}=\frac{N-1}{N} \sigma_{i, N}^{2}+\frac{N+1}{N^{2}}\left(x_{i, N+1}-\bar{x}_{i, N+1}\right)^{2}
\end{gathered}
$$

where $\bar{x}_{i, N}$ and $\sigma_{i, N}^{2}$ are the mean and the variance of the ith sample in the data window of length $N, \bar{x}_{i, N+1}$ and $\sigma_{i, N+1}^{2}$ are the online updated mean and variance of the $i$ th sample after the arrival of a new sample [19]. The data length $N$ of the moving window is very important for the computational efficiency, but till now, there is not an effective method to determine it. Currently, the setting of $N$ is totally depend on experience and experiment. In this work, we did many experiments and chose a proper $N$.

Then, the regression coefficient matrix of the PLS model can be updated online by using the updated mean and variance.

\subsection{Adaptive confidence limit setting}

The moving-window recursive algorithm can effectively solve the "data saturation" problem, and it can retain part of information of old samples through online updating the mean and variance of samples while tracking the dynamic change in the process, thus improving the prediction performance. The problem with the moving-window algorithm is the high model updating rate. Whenever a new sample is collected, the model will 
be updated, leading to a heavy computational load and reduced computational efficiency. To determine whether it is necessary to update the model, a confidence limit of the prediction error is used. A commonly used model updating method based on the confidence limit of prediction error is to set a small positive number $\delta$ as the confidence limit. When the prediction error is larger than $\delta$, the model will be updated. Otherwise, the model remains unchanged. Although this method can effectively reduce the model updating rate, it is difficult to set the confidence limit, which can balance between the prediction accuracy and the updating rate. Currently, the setting is totally dependent on experience or experiment. After the confidence limit is set, it remains constant throughout the whole process. Therefore, it cannot reflect the dynamic change and characteristics of the process. In this paper, an adaptive confidence limit, which is the standard deviation of prediction errors of the objective variables, is used as the criteria to determine whether the model should be updated [4, 20], as defined by

$$
\delta_{e}=\sqrt{\frac{\sum_{j=1}^{l} \sum_{i=1}^{N}\left(y_{i j}-\hat{y}_{i j}\right)^{2}}{\left(N-a-A_{0}\right) l}}
$$

where $a$ is the number of latent variables, $l$ is the number of output variables, $y_{i j}$ is the measured value for

the $i$ th measurement of the $j$ th output variable, $\hat{y}_{i j}$ is the corresponding predicted value given by the model, $A_{0}$ is set to be 1 if the model is standardised, otherwise $0 ; N$ is the number of modelling samples, that is the length of the moving window. The value of $N$ can not only affect the prediction accuracy of the model, but also can affect the value of the confidence limit, so as to affect the updating rate of the model. Because there is not an effective method to determine the value of $N$, we comprehensively consider the model's prediction accuracy and update rate, and determine a suitable value of $N$ through experiments.

The difference between this confidence limit and the conventional confidence limit is that the confidence limit is a statistical variable associated with the process characteristics. It can update adaptively with the dynamic change in the process characteristics. Therefore, the difficulties with the conventional method in setting the confidence limit and no reflection of the dynamic changes, are resolved.

\section{Soft sensor modelling based on time-difference, moving-window, recursive PLS and adaptive model updating}

\subsection{The proposed method}

The soft sensor modelling method based on time-difference, moving-window, recursive PLS and adaptive model updating makes use of the time difference values of the input and output variables to build a moving-window, recursive PLS model, and makes use of a statistical variable, which can change adaptively with the dynamic characteristics of a process and the changes of the model's prediction accuracy, as the confidence limit to determine whether the model should be updated. The advantages of this method include

(1) improving the model's approximation accuracy to the nonlinear characteristics of industrial processes,

(2) enhancing the model's tracking capabilities to dynamic changes in industrial processes, 
(3) reducing the model's update rate,

(4) improving the model's computational efficiency.

This modeling method can be described in detail as: Select a modelling sample set, calculate the time difference values of the input and output variables and build a time-difference moving-window PLS model; According to the statistical characteristics of prediction errors of the modelling sample set, calculate the standard deviation of the prediction errors by equation (16), this calculation result will be the initial confidence limit; Incorporate the new sample into the modelling sample set whenever a new sample is collected and update the mean and variance of the new modelling sample set, then discard the oldest sample; Calculate the prediction error of the new sample after the prediction value of the new sample is obtained. If this prediction error is larger than the confidence limit, the model will be updated, and the confidence limit will be updated too. Otherwise the model will remain unchanged.

\subsection{Modelling steps of the soft sensor model based on time-difference, moving-window, recursive PLS and adaptive model updating}

The modelling steps of the soft sensor model based on time-difference, moving-window, recursive PLS and adaptive model updating are as follows.

Step 1: Determine the window length $N$ of sample data (note that the $N$ samples constitute the modelling sample set $\left.\left[\boldsymbol{X}_{1}(t), \boldsymbol{y}_{1}(t)\right]\right)$, and calculate the time difference values $\Delta \boldsymbol{X}(t)$ and $\Delta \boldsymbol{y}(t)$ of the samples in $\left[\boldsymbol{X}_{1}(t), \boldsymbol{y}_{1}(t)\right]$ according to equations (7) and (8);

Step 2: Standardise $\Delta \boldsymbol{X}(t)$ and $\Delta \boldsymbol{y}(t)$ to obtain the training sample set $[\boldsymbol{X}(t), \boldsymbol{y}(t)]$ and calculate the mean and variance of these $N$-1 samples;

Step 3: Build time-difference, moving-window, recursive PLS model using the sample set $[\boldsymbol{X}(t), \boldsymbol{y}(t)]$, to obtain the regression coefficients $\hat{\boldsymbol{\beta}}$, (note that the prediction value $\Delta \hat{\boldsymbol{y}}(t)$ of $\Delta \boldsymbol{y}(t)$ can be obtained by the equation $\Delta \hat{\boldsymbol{y}}(t)=\Delta \boldsymbol{X}(t) \hat{\boldsymbol{\beta}})$

Step 4: Calculate the standard deviation $\delta_{e}$ by equation (16), and use $\delta_{e}$ as the initial confidence limit of the model (note that according to $\hat{y}(t)=\boldsymbol{y}(t-i)+\Delta \hat{y}(t)$, the predicted value of the objective variable can be obtained);

Step 5: Incorporate the new sample into the sample set $\left[\boldsymbol{X}_{1}(t), \boldsymbol{y}_{1}(t)\right]$ when a new sample $\left[\boldsymbol{x}_{\text {new }}, y_{\text {new }}\right]$ is collected; and calculate the time difference value $\Delta \boldsymbol{x}_{\text {new }}(t)$ and $\Delta y_{\text {new }}(t)$ of the new sample by equations (7) and (8);

Step 6: Recursively update the means and variances of the modelling sample set $\left[\boldsymbol{X}_{1}(t), \boldsymbol{y}_{1}(t)\right]$ and discard the oldest sample;

Step 7: According to $\Delta \hat{y}_{\text {new }}(t)=\Delta \boldsymbol{x}_{\text {new }}(t) \hat{\boldsymbol{\beta}}$, and $\hat{\boldsymbol{y}}_{\text {new }}(t)=\boldsymbol{y}(t-i)+\Delta \hat{y}_{\text {new }}(t)$, the prediction value $\hat{\boldsymbol{y}}_{\text {new }}(t)$ of the new sample can be obtained; 
Step 8: Calculate the root means squared (RMS) error;

Step 9: If the condition of RMS $>\delta_{e}$ is satisfied, then go to Step 3. Otherwise, go to Step 5.

\section{Industrial application}

\subsection{Process}

In a purified terephthalic acid (PTA) production process, 4-carboxy-benz-aldehyde (CBA) content reflects the process of oxidation. 4-CBA is one of the main by-products in the oxidation process, and is one of the important quality indicators. According to the studies on the reaction mechanism, if the 4-CBA content is too high, the oxidation reaction is insufficient and the p-xylene (PX) conversion rate is too low, which must be controlled in the production process. If the 4-CBA content is too low, per oxidation and the side reaction is increased, leading to the increase in consumption of energy, acetic acid and PX. To save energy and ensure the purity of PTA, the 4-CBA content must be controlled within a certain range. Therefore, it is important to implement online control of 4-CBA content. However, currently the 4-CBA content cannot be measured on-line. An alternative method is to use a soft sensor.

The detail of the PTA oxidation reaction process can be found in [21]. According to analysis on the process and oxidation reaction mechanism, 10 parameters are selected as the auxiliary variables (i.e. input variables). The objective variable (i.e. output variable) is the 4-CBA content in terephthalic acid (TA). The input and output variables of the soft sensor model are listed in Table 1.

\begin{tabular}{lll}
\multicolumn{3}{c}{ Table 1 Input and output variables of the model } \\
\hline Attribution & No. & \multicolumn{1}{c}{ Description } \\
\hline variables & 1 & Mixing tank feed flow \\
& 2 & Oxidation reactor feed flow \\
& 3 & Catalyst concentration \\
& 4 & Height of reactor liquid \\
& 5 & Reactor temperature \\
& 6 & Last oxygen content of reactor \\
& 7 & Discharge water of condenser 1 \\
& 8 & Discharge water of condenser 2 \\
& 9 & Meld temperature \\
& 10 & Last oxygen content of mould \\
& 11 & 4-CBA content \\
output & & \\
variable & &
\end{tabular}

\subsection{Results and discussion}

Because the 4-CBA content cannot be measured online, it is usually sampled three times each day and measured manually, so, only three samples per day can be used to build the soft sensor model. A large amount of process data were collected from a PTA oxidation reactor of a chemical fibre factory. After pre-processing, a data set with 231 samples was obtained. The data set is divided into two sets. One with 20 samples is used as a 
modelling sample set, and the other with 211 samples as a testing sample set to evaluate the performance of the model.

To verify the effectiveness of the proposed method, different models were used, including

(1) a PLS model

(2) a moving-window, recursive PLS model

(3) a time-difference, moving-window, recursive PLS model

(4) an adaptive model updating, moving-window, time-difference, recursive PLS model.

Their prediction results were compared and the models' prediction accuracy was evaluated by three indicators: (1) the maximum relative error, (2) the minimum relative error and (3) the RMS error. Each model uses 20 modelling samples (or the moving-window length), and 4 latent variables.
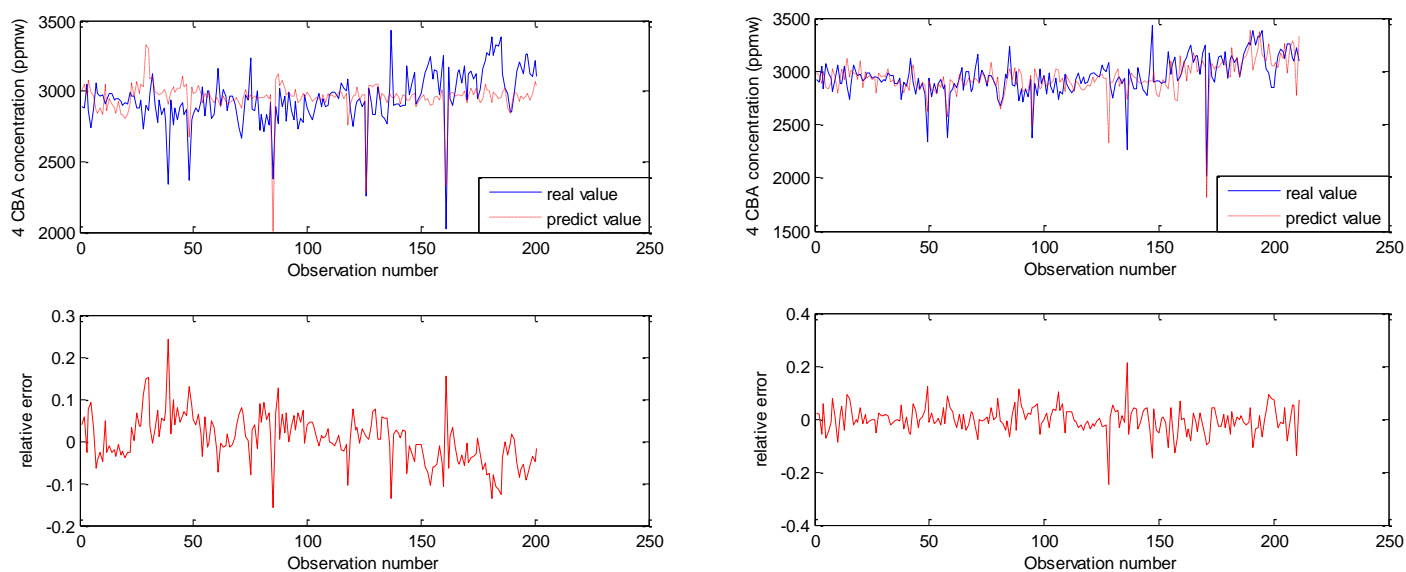

(a) PLS model

(b) Moving-window recursive PLS model
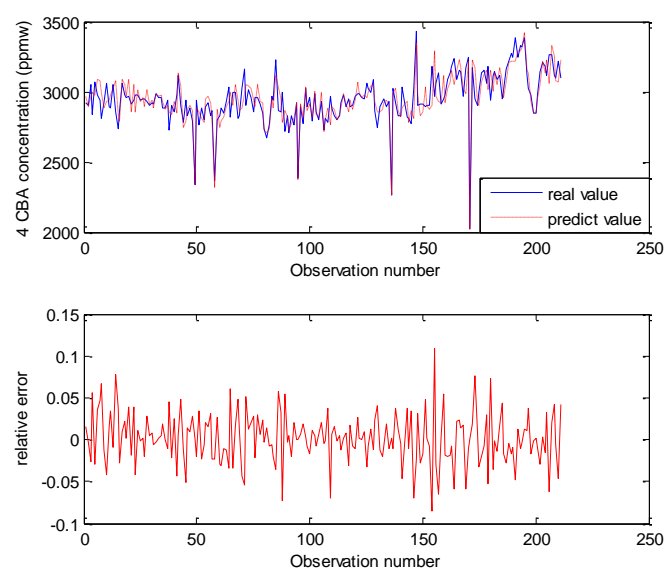

(c) Time-difference moving-window recursive PLS model

Figure 1 Measured values and predicted values with different models

To compare the prediction accuracy of the models, the comparison of the prediction results of the models and the measured values are shown in Figure 1. It can be seen that the coincidence of the prediction values of the PLS model and the measured values is poor. In particular, when the data change intensely, the error is large. Because the PLS algorithm is a linear algorithm, its regression accuracy to a nonlinear process is poor. The 
prediction results of the moving-window, recursive PLS model are much better than the PLS model, because the moving-window, recursive PLS model used can update its means and variances online, and it can track the dynamic characteristics of the process, thus enhancing the modelling accuracy. The prediction results of the time-difference, moving-window, recursive PLS model are the best, because this model uses the time difference values between the input and output variables to build the soft sensor model online, and it can improve the model's approximation accuracy to the nonlinear process, enhancing the prediction accuracy.

Comparing Figure 1 (a), (b) and (c), it can be seen that the time-difference, moving-window, recursive PLS algorithm is effective. It can greatly reduce the prediction errors, and the curve of prediction values is consistent with the curve of the measured values. But because the online updating method used in time-difference, moving-window, recursive PLS model updates the model's parameters whenever a new sample is collected, although this method can improve the prediction accuracy, it is at the expense of computational efficiency.

The prediction results of adaptive updating, time-difference, moving-window, recursive PLS model are shown in Figure 2. It can be seen that the prediction accuracy of adaptive updating, time-difference, moving-window, recursive PLS model is slightly lower than the time-difference, moving-window, recursive PLS model. But overall, the improvement is obvious compared with the ordinary moving-window, recursive PLS model.

Think of the computational efficiency, we can use model updating rate to compare it. The ordinary moving-window, recursive PLS model and the time-difference, moving-window, recursive PLS model updated whenever a new sample is collected. From Table 2, we can see that the number of updates is 211 . The adaptive model updating, time-difference, moving-window, recursive PLS model is only updated 157 times, the computational cost saving is about $25.6 \%$, and so the computational efficiency of the adaptive updating, time-difference, moving-window, recursive PLS model has been greatly improved. It can be concluded that the adaptive model updating, time-difference, moving-window, recursive PLS model proposed in this paper is effective as it can not only improve the prediction accuracy of the model, but also improve the computational efficiency.
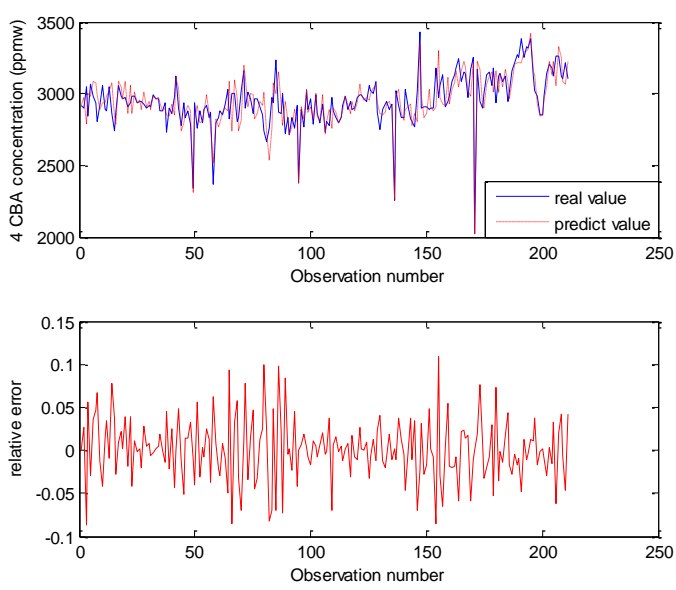

Figure 2 Real values and the predict values of adaptive updating time-difference moving-window recursive PLS model 
Table 2 Performance comparison of different models for modelling 4-CBA content

\begin{tabular}{|l|c|c|c|c|}
\hline \multicolumn{1}{|c|}{ Model } & $\begin{array}{c}\text { Maximum } \\
\text { relative error }\end{array}$ & $\begin{array}{c}\text { Minimum } \\
\text { relative error }\end{array}$ & $\begin{array}{c}\text { Root mean } \\
\text { square error }\end{array}$ & $\begin{array}{c}\text { Model } \\
\text { updating rate }\end{array}$ \\
\hline PLS & 0.261 & -0.191 & 0.0586 & 0 \\
\hline Moving-window, recursive PLS & 0.196 & -0.271 & 0.0517 & 211 \\
\hline Time-difference, moving-window, recursive PLS & 0.110 & -0.0859 & 0.0289 & 211 \\
\hline $\begin{array}{l}\text { Adaptive model updating, time-difference, } \\
\text { moving-window, recursive PLS }\end{array}$ & 0.110 & -0.0869 & 0.0342 & 157 \\
\hline
\end{tabular}

Table 3 Overall qualitative comparison of each model

\begin{tabular}{|l|c|c|c|}
\hline \multicolumn{1}{|c|}{ Model } & Prediction accuracy & Algorithmic complexity & Calculating time \\
\hline PLS & Low & Low & Short \\
\hline Moving-window, recursive PLS & Middle & Middle & Long \\
\hline Time-difference, moving-window, recursive PLS & High & High & Long \\
\hline $\begin{array}{l}\text { Adaptive model updating, time-difference, } \\
\text { moving-window, recursive PLS }\end{array}$ & High & High & Middle \\
\hline
\end{tabular}

The performance of different models for 4-CBA content is compared in Table 2, including the maximum relative error, the minimum relative error, the root mean square error of prediction, and the model updating rate. It can be seen that the prediction accuracy of the time-difference, moving-window, recursive PLS model has been greatly improved, but its computational efficiency is also low. The computational efficiency of the adaptive model updating, time-difference, moving-window, recursive PLS model has been greatly improved with a little loss of prediction accuracy. The overall qualitative comparison of each model is given in Table 3, including prediction accuracy, algorithmic complexity and calculating time.

From Fig.1, Fig.2 and Table 2, Table3, we can see that the proposed method has high prediction accuracy and high computational efficiency. Because this method uses time difference moving-window recursive PLS model, it can not only fully retain the good characteristics of linear PLS model, but also improve the model's prediction accuracy to nonlinear process. Furthermore, this method uses adaptive confidence limit that can update adaptively with the time-varying nature of the process to reduce the model updating rate, so to improve the computational efficiency.

\section{Conclusions}

The ordinary PLS algorithm is popular in soft sensor modelling, but cannot reflect the nonlinear relationship between the input and output variables. Although the recursive PLS algorithm can reflect the nonlinear relationship to some extent, the model updating rate is high, and hence the computational efficiency is low. To deal with these problems, a soft sensor modelling method based on time-difference, moving-window, recursive PLS and adaptive model updating is proposed in this paper. This method firstly uses the time difference values between the input and output variables to build a time-difference moving-window recursive PLS model. Then a confidence limit is generated automatically based on the initial characteristics of the process, which can be updated adaptively with the time-varying nature of the process and the prediction accuracy of the model. 
To verify the method, the proposed method was used to predict the 4-CBA content in a PTA oxidation reaction process. The results show that the soft sensor modelling method based on the time-difference, moving-window, recursive PLS and adaptive model updating can not only improve the prediction accuracy significantly, but also greatly improve the computational efficiency.

In industry, many process variables are difficult to measure online and the processes have time-variant and nonlinear characteristics. The soft sensor method proposed in this paper is an effective alternative choice to implement the real-time estimation of these process variables, thus making online quality control, advanced control and optimal control of these processes possible.

\section{Acknowledgements}

The authors would like to thank the National Natural Science Foundation of China for supporting this work (Grant No. 61304125, 61203133 and 61304136) and the Open Research Project of the State Key Laboratory of Industrial Control Technology, Zhejiang University, China (Grant No. ICT1600265). Yongfeng Fu would also like to thank the Chinese Scholarship Council for supporting her to be an academic visitor at The University of Manchester in the UK.

\section{References}

[1] KANO M and NAKAGAWA Y 2008 Data-based process control, and quality improvement: recent developments and applications in steel industry Comput. Chem. Eng. 32 12-24

[2] KADLEC P, GABRYS B and STRANDT S 2009 Data-driven soft sensors in process industry Comput. \& Chem. Eng. 33 795-814

[3] FORTUNA L, GRAZIANI S, RIZZO A and XIBILIA M G 2007 Soft sensors for monitoring and control of industrial processes (Berlin: Springer-Verlag)

[4] XU O G, CHEN X H, FU Y F and LI L J 2014 Recursive PLS modeling based on model performance assessment and its application CIESC J., 65 4875-4882

[5] WANG C P, YU Z J and MENG F Q 2013 Discount moving window recursive PLS algorithm and its application to process of polypropylene production CIESC J. 64 4592-4598

[6] LIU J, CHEN D and SHEN J 2010 Development of self-validating soft sensors using fast moving window partial least squares Indus. \& Eng. Chem. Res. 49 11530-11546

[7] ROSIPAL R 2003 Kernel partial least squares for nonlinear regression and discrimination Neural Network World 13 291-300.

[8] ROSIPAL R and TREJO L J 2002 Kernel partial least squares regression in reproducing kernel Hilbert space J. of Machine Learning Res. 02 97-123

[9] HELLAND K, BERNTSEN H E, BORGEN O S and MARTENS H 1992 Recursive algorithm for partial least squares regression Chemometrics \& Intelligent Lab. System 14 129-137

[10] QIN S J 1998 Recursive PLS algorithms for adaptive data modeling Compt. Chem. Eng. 22 503-514

[11] SONG K, WANG H Q and LI P 2004 Discounted-measurement RPLS algorithm and its application to quality control of the rubber mixing process CIESC J. 55 942-946

[12] MU S J, ZENG Y Z, LIU R L, WU P, SU H Y and CHU J 2006 Online dual updating with recursive PLS model and its application in predicting crystal size of purified terephthalic acid (PTA) process J. of Process Control 16 557-566

[13] NI W, TAN S K, NG W J and BROWN S D 2012 Moving-window GPR for nonlinear dynamic system modeling with dual updating and dual preprocessing Indus. \& Eng. Chem. Res. 51 6416-6428

[14] GELADI P and KOWALSKI B R 1986 Partial least squares regression: a tutorial Analytica Chimica Acta 185 1-17

[15] KANEKO H and FUNATSU K 2011 A soft sensor method based on values predicted from multiple intervals of time difference for improvement and estimation of prediction accuracy Chemometrics and Intelligent Lab. Systems 109 197-206

[16] KANEKO H and FUNATSU K 2011 Maintenance-free soft sensor models with time difference of process variables 
Chemometrics and Intelligent Lab. Systems 107 312-317

[17] RUAN H M, TIAN X M and WANG P 2013 Soft measurement modeling method based on time difference PLS with time delay estimation Automation in Petro-chem. Industry 49 35-39.

[18] KADLEC P, GRBI R and GABRYS B 2011 Review of adaptation mechanisms for data-driven soft sensors Comput. \& Chem. Eng. 35 1-24

[19] FU Y F, SU H Y and CHU J 2007 MIMO soft-sensor model of nutrient content for compound fertilizer based on hybrid modeling technique Chinese J. of Chem. Eng. 15 554-559

[20] LEE Y, KIM M, CHU Y and HAN C 2005 Adaptive multivariate regression modeling based on model performance assessment Chemometrics \& Intelligent Lab. Systems 78 63-73

[21] LIU R L 2004 Some studies on soft sensor technology and their applications to industry process PhD Thesis Zhejiang University 